\title{
Computation of Trailing Edge Noise with a Discontinuous Galerkin Method
}

\author{
M. Bauer \\ DLR (German Aerospace Center), Institute of Aerodynamics and Flow \\ Technology, Lilienthalplatz 7, 38108 Braunschweig, Germany \\ marcus.bauer@dlr.de
}

\section{Summary}

Trailing edge noise of a semi-infinite, thin, flat plate situated in low Mach number flow is computed in two spatial dimensions. The Acoustic Perturbation Equations (APE), which are employed as governing equations, are discretized via a Discontinuous Galerkin Method (DGM). Results are compared with theory and Finite Difference (FD) computations. Next to the radiated sound field, special attention is paid to the conditions very close to the trailing edge (TE).

\section{Introduction}

Airframe noise is generated by the transformation of turbulent energy into acoustic energy in the vicinity of edges, such as the TE of an airfoil or of a deployed slat. Considering the low noise engines of modern airliners, it is an important noise source during the approach phase. Unstructured grids greatly ease the mesh generation process when airframe noise of complex geometries is of interest. The DGM $[1,2,6,7,11]$ provides a robust, high-order accurate discretization even on this type of grid and offers excellent wave propagation characteristics. However, the fidelity of the computed airframe noise field also depends on the fidelity of the computed aeroacoustic source mechanism: the aforementioned transformation of turbulent energy into acoustic energy at edges.

References $[6,11]$ investigate the reliability of the DGM to compute the acoustic response of various two-dimensional (2D) objects to incident vortical gusts. An infinitely thin, flat plate and a non-lifting as well as a lifting airfoil served as test objects, and the nonlinear Euler equations as well as the linearized Euler equations were considered as governing equations. Results are in good or very good agreement with semi-analytical and numerical reference data.

In the present work, TE noise of a semi-infinite, thin, flat plate is computed with a DGM on an unstructured, triangular grid (2D). The APE $[8,9]$ are employed as governing equations. To simplify matters, the flow has low 
Mach number, and the turbulent source term of the APE is computed from a simple, analytical velocity field. DG-APE results are compared to theoretical solutions and to FD computations.

The goal of this work was to figure out, how reliably the employed DGM captures aeroacoustic noise generation at a TE.

\section{Theory}

\subsection{Particularly employed form of APE}

The APE $[8,9]$ for constant sound speed $c_{0}$ read in matrix-vector notation:

$$
\frac{\partial \boldsymbol{U}}{\partial t}+\frac{\partial \boldsymbol{F}^{x}}{\partial x}+\frac{\partial \boldsymbol{F}^{y}}{\partial y}-\boldsymbol{S}=\mathbf{0} .
$$

In (1), $t$ denotes time, and $x$ and $y$ are the two-dimensional Cartesian spatial coordinates. $\boldsymbol{U}$ is the vector of unknowns and $\boldsymbol{F}^{x}=\boldsymbol{A} \boldsymbol{U}$ and $\boldsymbol{F}^{y}=\boldsymbol{B} \boldsymbol{U}$ are the flux vectors with

$$
\boldsymbol{U}=\left(\begin{array}{l}
p^{\prime} \\
u^{\prime} \\
v^{\prime}
\end{array}\right), \quad \boldsymbol{A}=\left(\begin{array}{ccc}
u_{0} & \varrho_{0} c_{0}^{2} & 0 \\
\frac{1}{\varrho_{0}} & u_{0} & v_{0} \\
0 & 0 & 0
\end{array}\right), \quad \boldsymbol{B}=\left(\begin{array}{ccc}
v_{0} & 0 & \varrho_{0} c_{0}^{2} \\
0 & 0 & 0 \\
\frac{1}{\varrho_{0}} & u_{0} & v_{0}
\end{array}\right),
$$

where $p$ denotes pressure, $\varrho$ density, and $u$ and $v$ the $x$ - and $y$-directed velocity component, respectively. A prime marks unknown time- and space-dependent perturbation variables, whereas an index 0 indicates time-averaged, i.e. steady, mean flow quantities known in advance. The computations presented in this article are based on a simple, spatially constant mean flow field, see section 3.1 for details.

$\boldsymbol{S}$ is the APE source vector. For vortex sound problems like airframe noise, its first component can be assumed to be zero, and its second and third component are given by the linear perturbed Lamb-vector $[8,9]$. In 2D Cartesian coordinates, the employed source vector thus reads

$$
\boldsymbol{S}=-\left(\begin{array}{c}
0 \\
-\omega_{3}^{\prime} \cdot v_{0}-\omega_{30} \cdot v^{\prime} \\
\omega_{3}^{\prime} \cdot u_{0}+\omega_{30} \cdot u^{\prime}
\end{array}\right)
$$

where $\omega_{3}=\frac{\partial v}{\partial x}-\frac{\partial u}{\partial y}$ is the $z$-component of the vorticity vector.

\subsection{Discretization of APE via DGM}

The 2D computational domain is partitioned into non-overlapping triangular elements $E^{(m)}$. The entries of the vectors $\boldsymbol{U}, \boldsymbol{F}^{x}, \boldsymbol{F}^{y}$, and $\boldsymbol{S}$ are approximated by an expansion of type

$$
f^{(m)} \approx \tilde{f}^{(m)}=\sum_{l=1}^{N} \hat{f}_{l}^{(m)}(t) \cdot \Phi_{l}^{(m)}(x, y)
$$


in each element. As the approximation $\tilde{f}^{(m)}$ is supposed to be a full $2 \mathrm{D}$ polynomial of degree three in this work, the number $N$ of expansion terms according to the Pascal triangle is $N=10$ [14]. The symbols $\hat{f}_{l}^{(m)}$ denote the expansion coefficients and $\Phi_{l}^{(m)}$ are given spatial shape functions. The overall approximate solution $\tilde{\boldsymbol{U}}$ may be discontinuous along the edges between neighboring elements in the framework of the DGM. A detailed description of the particularly employed DGM may be found in Ref. [2].

It was implemented in the programming language FORTRAN 90/95, using as reference shape-functions Lagrange polynomials of degree three with standard distribution of the so-called nodes $[2,14]$. Consistently, time integration is performed by a standard explicit fourth order Runge-Kutta scheme, and the overall order of accuracy of the code is four, which was verified by convergence tests.

\section{Computations}

\subsection{Test Setup}

Dimensionless quantities were used. They were computed from dimensional quantities, marked by superscript $*$ or subscript $\infty$, like

$$
t=t^{*} \frac{c_{\infty}}{L^{*}}, \quad \boldsymbol{x}=\frac{\boldsymbol{x}^{*}}{L^{*}}, \quad \varrho=\frac{\varrho^{*}}{\varrho_{\infty}}, \quad \boldsymbol{v}=\frac{\boldsymbol{v}^{*}}{c_{\infty}}, \quad p=\frac{p^{*}}{\varrho_{\infty} c_{\infty}^{2}},
$$

where $\boldsymbol{x}=[x, y]^{\mathrm{T}}$ is the coordinate vector and $\boldsymbol{v}=[u, v]^{\mathrm{T}}$ the velocity vector. The dimensional reference quantities are a length of $L^{*}=1 \mathrm{~m}$ as well as the density and sound speed at infinity.

Actually, TE noise of an infinitely thin, flat plate with a finite length of $0.2 \mathrm{~m}$ was calculated. Conditions of a semi-infinite plate were emulated by the fact, that the computational domain only comprised the plate's rear part, see figure 1 .

To approximate low Mach number flow, the entries of the APE mean flow matrices $\boldsymbol{A}$ and $\boldsymbol{B}$ were set to $u_{0}=v_{0}=0$ and $\varrho_{0}=c_{0}=1$.

A simple, analytical, turbulent velocity field $\boldsymbol{v}_{t}=\left[0, \cos \left(\alpha x-\alpha v_{c} t\right)\right]^{\mathrm{T}}$, with wave number $\alpha=1571.0$ and convection velocity $v_{c}=0.05$, was substituted for the velocity perturbations of the source vector $\boldsymbol{S}$ from Eq. (3). This velocity field is a simplified form of the synthetic turbulent velocity field as proposed in the framework of the SNGR (Stochastic Noise Generation and Radiation) method [3, 4], where it is calculated as the sum of some ten or hundred discrete, random Fourier modes. Note, that $\boldsymbol{v}_{t}$ is frozen and incompressible, $\nabla \cdot \boldsymbol{v}_{t}=0$. The source was computed in a single source patch with extension $-0.036 \leq x \leq 0.036,0.0 \leq y \leq 0.004$ above the $\mathrm{TE}$, see also figure 1. The final source vector $\boldsymbol{S}$ follows from Eq. (3) by furthermore assuming 
$u_{0}=v_{c} \neq 0, v_{0}=0$ and by introducing additional weighting functions $W_{i}$ :

$$
\boldsymbol{S}=-W_{a}(x) \cdot W_{b}(y) \cdot W_{t}(t) \cdot\left(\begin{array}{c}
0 \\
0 \\
v_{c} \frac{\partial v_{t y}}{\partial x}
\end{array}\right) .
$$

The weighting functions all ranged between zero and one. To reduce spurious noise $[3,8], W_{a}(x)$ smoothly faded the source term in and out in $x$ direction, i.e., the direction of convection of $\boldsymbol{v}_{t} . W_{b}(y)$ modeled the generic $y$-distribution of the turbulent kinetic energy at the TE of a flat plate [3]. The source was faded in temporally at the beginning of a computation by $W_{t}(t)$ to attenuate spurious steady contributions to the perturbation quantities in the source patch. Such steady contributions actually do not cause any spurious noise, but they corrupt contour plots.

Comparative computations were performed with DLR's CAA $^{1}$ code PIANO $^{2}$ [5], which employs the fourth order accurate Dispersion Relation Preserving (DRP) FD scheme [12] for the spatial discretization. Artificial selective damping (ASD) [13] damped spurious short waves. The standard fourth order explicit Runge Kutta scheme was used for time integration in PIANO, too, just like in the DG-APE code.

Tables 1 and 2 list important parameters of the block-structured PIANO grids and of the unstructured, triangular DG grids. In both cases, there was a coarse, a medium, and a fine mesh, and all meshes were refined in the source patch area. The triangular grids cover a circular domain with radius $r=0.1$ around the TE, whereas the block-structured grid domains are squares with $-0.1 \leq x, y \leq 0.1$.

\section{$3.2 \quad$ Results}

Figure 1 shows a snapshot of the pressure perturbations computed with the DG-APE code on the coarse grid. Small scale, large amplitude turbulent structures are induced in the source patch, and sound waves are radiated from the TE. They are perfectly symmetric (with opposite sign) along $y=0$, although there is only a source patch above the flat plate. Contour plots from the other computations are very similar.

Figure 2 illustrates root mean square values $\tilde{p}$ of the sound pressure at receiving point $(x, y)=(0,0.08)$ above the TE. Considering PIANO, the amplitude increases when the ASD damping coefficient is reduced. The DG amplitudes exceed those from PIANO, but the difference decreases towards finer grids. On the finest grids, $\tilde{p}$ from DG exceeds $\tilde{p}$ from PIANO (damping 0.03 ) by about $7 \%$ or $0.6 \mathrm{~dB}$. The amplitude differences between the various simulations do not develop on the way of the sound waves from the TE to the receiving point $(x, y)=(0,0.08)$. They are almost identical at e.g. a receiving

\footnotetext{
${ }^{1}$ Computational Aero Acoustics

${ }^{2}$ Perturbation Investigation of Aerodynamic Noise
} 
point $(x, y)=(0,0.01)$ much closer to the TE. Thus, they must be due to a different strength of the aeroacoustic noise generation mechanism at the TE.

The exact solution very close to the TE can be calculated analytically. Since $\boldsymbol{v}_{0}=\mathbf{0}, \varrho_{0}=$ const, and $\lim _{y \rightarrow 0} W_{b}(y)=0$, i.e., $\lim _{y \rightarrow 0} \boldsymbol{S}=\mathbf{0}$, the APE transform into the simple homogeneous acoustic wave equation, where the velocity potential $\Phi^{\prime}$ may be substituted for the pressure perturbations $p^{\prime}$ yielding $\frac{1}{c_{0}^{2}} \frac{\partial^{2} \Phi^{\prime}}{\partial t^{2}}-\Delta \Phi^{\prime}=0$. This further simplifies to the potential equation $\Delta \Phi^{\prime}=0$ in the vicinity of edges. The respective solution for a semi-infinite, thin, flat plate can thus be found via conformal mapping:

$$
\Phi^{\prime}=r^{0.5} \cos (\gamma / 2), \quad r \neq 0,
$$

with $r=\sqrt{x^{2}+y^{2}}$ and $\gamma=180^{\circ}-\theta$, see figure 3 (top left). Finally, one can calculate $\boldsymbol{v}^{\prime}=\nabla \Phi^{\prime}$, and e.g. end up with:

$$
v^{\prime}=0.5 r^{-1.5}(y \cos (\gamma / 2)+x \sin (\gamma / 2)), \quad r \neq 0,
$$

as illustrated qualitatively in figure 3, too. Solution (8) is not defined at the TE, i.e., at $r=0$. Furthermore, if an observer, who is initially located right at the TE, moves an infinitesimal distance in any direction, then $v^{\prime} \rightarrow \infty$, except along the surface of the plate, where $v^{\prime}=0$. Also note that $\nabla \cdot \boldsymbol{v}^{\prime}=$ $\nabla \cdot \nabla \Phi^{\prime}=\Delta \Phi^{\prime}=0$, i.e., theoretically no noise is generated right at the TE.

Figure 3 also shows snapshots of computational results for the $v^{\prime}$ field at the TE. The big shape on top of the respective pictures was induced by the source. The amplitude differences from figure 2 can be explained by way of figure 3 in terms of the structure which developed around the TE. It was always stronger in DG than in PIANO. Focusing on the DG solutions, its extension decreased the finer the grid (although its maximum amplitude increased). In the PIANO computations, the structure grew with reduced damping and towards finer grids. It may also be worth noting, that the DG solutions are closer to the difficult theoretical solution at the TE than the PIANO results, because -like in theory- the maximum occurred right at the TE in DG. It always occured somewhat downstream in PIANO, though, where a coarser grid and increased damping moved it further downstream.

Finally, figure 4 presents sound pressure directivities $\Gamma(\theta)=\frac{\tilde{p}(\theta)}{\tilde{p}(\theta)_{\max }}$. In spite of the difficult situation right at the TE, there are hardly any differences between the solutions from DG and PIANO. Furthermore, for a halved convection velocity of $v_{c}=0.025$, the theoretically expected cardioid curve $\Gamma(\theta)=\sin (\theta / 2)[10]$ is met very well.

\section{Conclusions}

The employed DG scheme seems capable of capturing aeroacoustic noise generation at the TE of a semi-infinite, thin, flat plate situated in low Mach 
number flow: the computed sound pressure field looks very reasonable, and there is excellent agreement of the sound pressure directivities from DG, FD, and theory. But, on the other hand, the sound pressure amplitudes slightly differ among the various computations. This is explained by the conditions very close to the TE, where the computations deviate from each other and also from the tough theoretical solution.

To judge the computed amplitudes, the exact solution for the whole domain may be calculated via the respective tailored Green's function. The influence of a non-zero, non-uniform mean-flow velocity field may be another point of future interest.

\section{References}

[1] H. L. Atkins and C.-W. Shu. Quadrature-Free Implementation of Discontinuous Galerkin Method for Hyperbolic Equations. AIAA Journal, 36(5):775-782, 1998.

[2] M. Bauer, J. Dierke, and R. Ewert. Application of a Discontinuous Galerkin Method to Predict Airframe Noise. AIAA-paper 2009-3175, American Institute for Aeronautics and Astronautics, 2009.

[3] M. Bauer, A. Zeibig, and P. Költzsch. Application of the SNGRModel to Compute Trailing Edge Noise. http://www.ias.et.tudresden.de/akustik/Publikationen/Stroemungsakustik/Publikationen_STAK.htm. Research Report, Institute of Acoustics and Speech Communication, Dresden University of Technology, Germany, 2006.

[4] W. Béchara, C. Bailly, P. Lafon, and S. Candel. Stochastic Approach to Noise Modeling for Free Turbulent Flows. AIAA Journal, 32(3):455-463, 1994.

[5] J. W. Delfs, M. Bauer, R. Ewert, H. A. Grogger, M. Lummer, and T. G. W. Lauke. Numerical Simulation of Aerodynamic Noise with DLR's Aeroacoustic Code PIANO (Version 5.1). Manual, Deutsches Zentrum für Luft- und Raumfahrt e.V., Institute of Aerodynamics and Flow Technology, 2007.

[6] M. Dumbser. Arbitrary High Order Schemes for the Solution of Hyperbolic Conservation Laws in Complex Domains. PhD thesis, University of Stuttgart, Germany, 2005.

[7] K. Ehrenfried, C. Meyer, and A. Dillmann. Simulation of Sound Propagation in Boundary Layers based on Möhring's Acoustic Analogy. AIAA-paper 20033272, American Institute for Aeronautics and Astronautics, 2003.

[8] R. Ewert, M. Meinke, and W. Schröder. Computation of Trailing Edge Noise via LES and Acoustic Perturbation Equations. AIAA-paper 2002-2467, American Institute for Aeronautics and Astronautics, 2002.

[9] R. Ewert and W. Schröder. Acoustic Perturbation Equations based on Flow Decomposition via Source Filtering. Journal of Computational Physics, 188(2):365-398, 2003.

[10] J. E. Ffowcs Williams and L. H. Hall. Aerodynamic Sound Generation by Turbulent Flow in the Vicinity of a Scattering Half Plane. Journal of Fluid Mechanics, 40(4):657-670, 1970.

[11] P. Rasetarinera, D. A. Kopriva, and M. Y. Hussaini. Discontinuous Spectral Element Solution of Acoustic Radiation from Thin Airfoils. AIAA Journal, 39(11):2070-2075, 2001. 
[12] C. K. W. Tam and J. C. Webb. Dispersion-Relation-Preserving Finite Difference Schemes for Computational Acoustics. Journal of Computational Physics, 107:262-281, 1993.

[13] C. K. W. Tam, J. C. Webb, and Z. Dong. A Study of the Short Wave Components in Computational Acoustics. Journal of Computational Acoustics, 1:1-30, 1993.

[14] O. C. Zienkiewicz, R. L. Taylor, and J. Z. Zhu. The Finite Element Method - Its Basis and Fundamentals. Elsevier Butterworth-Heinemann, 6th Edition, 2005 .

Table 1 Parameters (dimensionless) of block-structured grids; $\Delta$ denotes distance between grid points; $\Delta_{\min }=\Delta x_{\min }=$ $\Delta y_{\min }$, and $\Delta_{\max }=\Delta x_{\max }=\Delta y_{\max }$

\begin{tabular}{|r|c|c|c|}
\hline & coarse & medium & fine \\
\hline$\Delta_{\min } \cdot 1000$ & 0.12 & 0.08 & 0.06 \\
$\Delta_{\max } \cdot 1000$ & 5.00 & 3.33 & 2.50 \\
no. of pts. & 161.102 & 355.810 & 630.872 \\
\hline
\end{tabular}

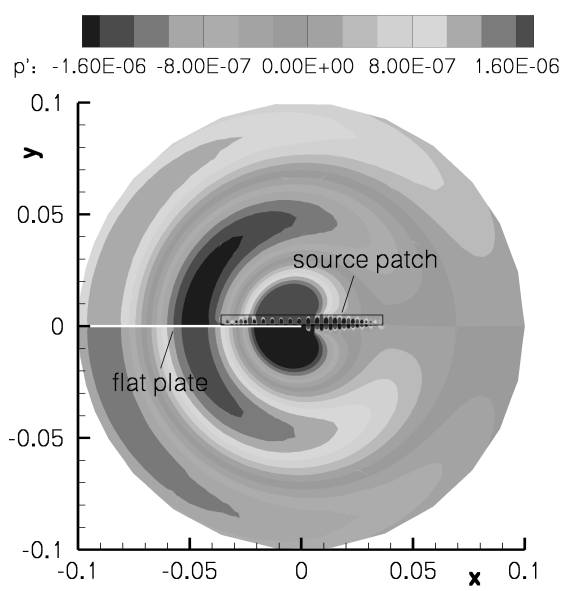

Figure 1 Snapshot of pressure perturbations $p^{\prime}$ from DG-APE code on coarse triangular grid.
Table 2 Parameters (dimensionless) of unstructured grids; $L$ denotes triangle edge length, $N=10$ is the number of shape functions per element according to (4), and $E$ the overall number of elements.

\begin{tabular}{|r|c|c|c|}
\hline & coarse & medium & fine \\
\hline$L_{\min } \cdot 1000$ & $\approx 0.500$ & $\approx 0.375$ & $\approx 0.300$ \\
$L_{\max } \cdot 1000$ & $\approx 13.33$ & $\approx 10.00$ & $\approx 8.00$ \\
$N \cdot E$ & 94.600 & 174.880 & 268.720 \\
\hline
\end{tabular}

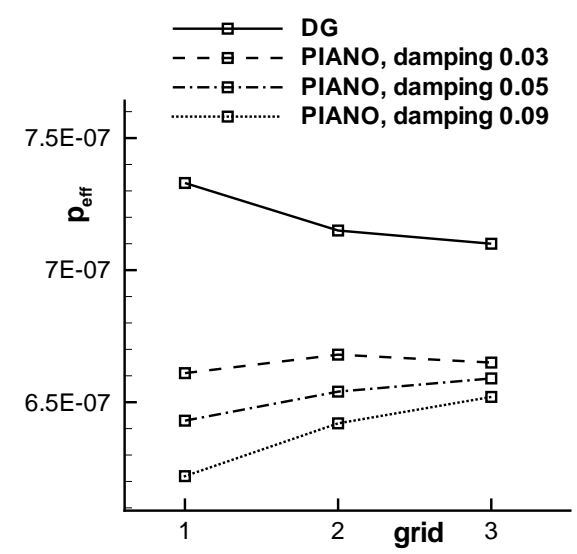

Figure 2 Root mean square values of sound pressure at $(x, y)=(0,0.08)$; grid numbers 1, 2 and 3 denote coarse, medium and fine grid, respectively. 

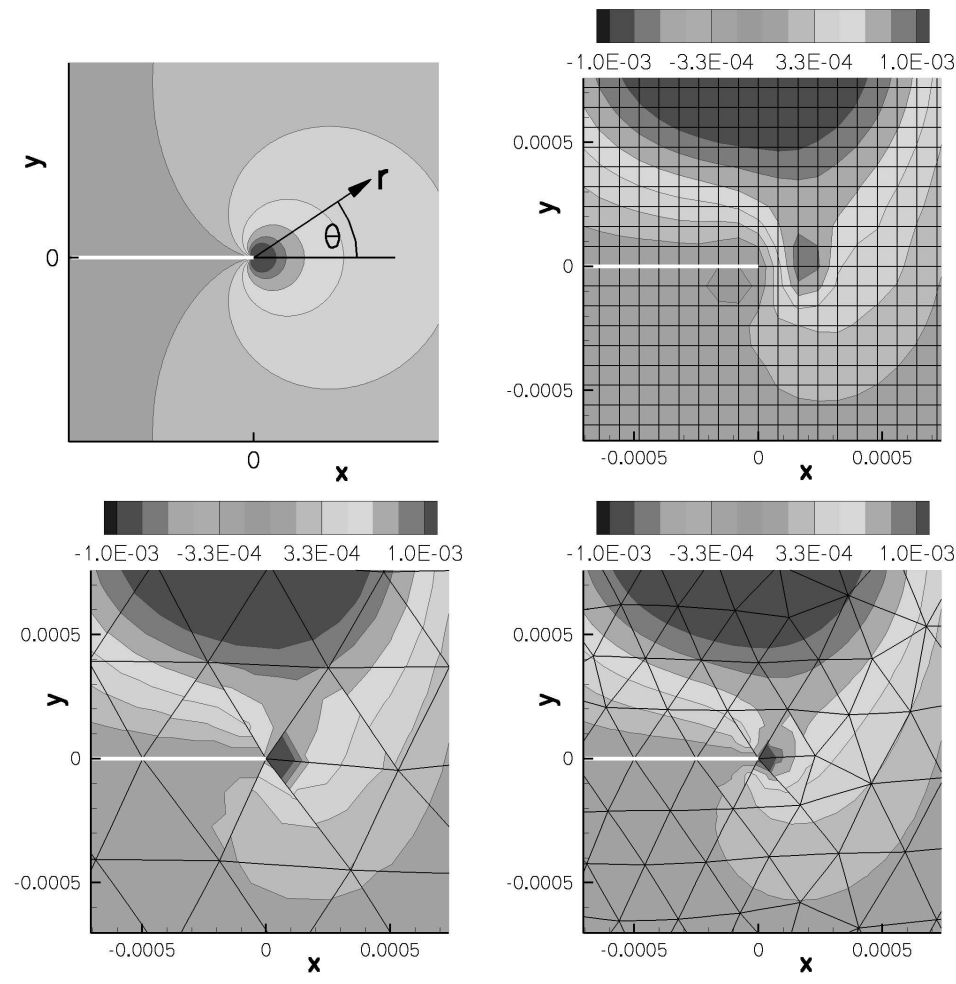

Figure 3 Solutions of $v^{\prime}$ field in vicinity of flat plate trailing edge; top left: theory; top right: PIANO with damping 0.05 on medium grid; bottom left: DG on coarse grid; bottom right: DG on fine grid.
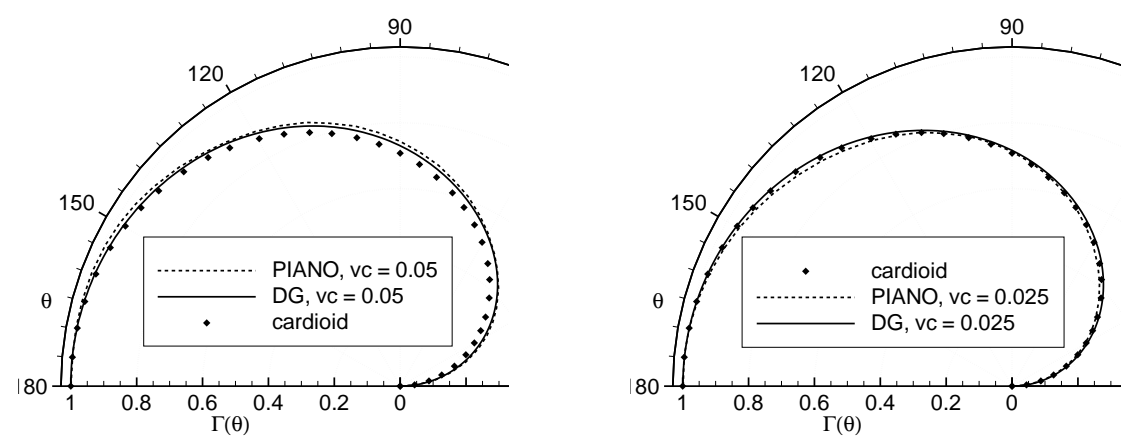

Figure 4 Sound pressure directivities along circle with dimensionless radius $r=0.08$ around flat plate trailing edge using medium grids and damping 0.03 within PIANO. 